\title{
PULMONARY INVOLVEMENT IN PRIMARY SJÖGREN'S SYNDROME: RESULTS FROM A PORTUGUESE TERTIARY CENTER
}

\author{
Ana Martins ${ }^{1, \star}$, Sofia Pimenta ${ }^{1}$, Lúcia Costa ${ }^{1}$ \\ 1.Centro Hospitalar Universitário de São João, Porto, Portugal. \\ *Corresponding author: anaigmartins.med@gmail.com
}

\section{BACKGROUND}

Primary Sjögren's syndrome (pSS) is a chronic systemic autoimmune disease. Pulmonary manifestations are among the most frequent extraglandular manifestations and include interstitial lung disease (ILD), which is an important factor contributing to impaired quality of life and survival rate of pSS patients.

\section{METHODS}

We performed a retrospective analysis of patients with pSS who fulfilled the ACR/EULAR 2016 classification criteria followed in our university hospital. Demographic data, disease duration, extraglandular involvement and histopathologic data were collected and analyzed. The European League Against Rheumatism (EULAR) Sjögren's syndrome disease activity index (ESSDAI) score was calculated. Fisher's exact test/chi-squared test and the Mann-Whitney $U$ test were used to compare the groups with and without pulmonary involvement (PI). In patients with PI, clinical characteristics, pulmonary function tests with diffusing capacity for carbon monoxide (DLCO), 6-minute-walk test, histopathologic patterns and treatment were collected from medical records. Paired samples t-test was used to compare patients at diagnosis onset and at the last evaluation. P-value $<0.05$ was considered statistically significant.

\section{RESULTS}

In total, 66 patients were included. PI was documented in 8 patients (12.1\%) and disease duration was 3.5 (1.5-7.25) years. Patients with PI were older ( $p=0.027)$ and had a higher ESSDAI score $(p<0.001)$ than patients without PI. No other statistically significant difference was found. In patients with PI $(n=8), 7$ had chronic cough and 6 had dyspnea. Pulmonary arterial hypertension was found in 1 patient (12.5\%). Four patients had usual interstitial pneumonia, 2 had lymphocytic interstitial pneumonia, 1 had nonspecific interstitial pneumonia and 1 had pleuroparenchymal fibroelastosis. Spirometry at diagnosis found a restrictive pattern in 3 patients, obstructive pattern in 3 patients and was normal in 2 patients. Comparing the values of spirometry at diagnosis and at the last evaluation (time interval 3.5 years), a quick decrease of DLCO (decreased by $28 \%$ ), with a significant difference between the 2 evaluations ( $p=0.021$ ) was found. Also, a decline in pulmonary function (forced vital capacity decreased by $8.8 \%$ and forced expiratory volume in 1 second decreased by 6.0\%) was found, but without significant difference. Functional test also shown a quick deterioration, with a significant decrease in the walk distance $(p=0.025)$ and a significant decrease in oxygen saturation during exercise $(p=0.018)$.

\section{CONCLUSION}

Interstitial lung disease is a frequent manifestation in pSS patients. In this study, patients with PI were older and had more severe disease. As documented in this study, PI in pSS is a serious condition and has a quick progression, with rapid function and functional impairment. An early diagnosis and an adequate treatment are essential to guarantee a better prognosis.

\section{KEYWORDS}

Sjögren's syndrome, pulmonary involvement, pulmonary function tests. 